\title{
Fractal Pattern Formed in Ag-In Electrodeposits
}

\author{
M. Saitou*, H. Ota and S.M. Asadul Hossain \\ University of the Ryukyus, Department of Mechanical Systems Engineering, 1 Senbaru Nishihara, Okinawa, 903-0213, \\ Japan
}

\begin{abstract}
We investigated pattern formation in Ag-In electrodeposition by constructing a morphology diagram that indicates the presence of pattern at a current density and cathode potential. The patterns that emerge as a fishbone and tree are found to have a fractal property. As the current density increases, the fishbone-like pattern with a fractal dimension of 1.44 changes into the tree-like pattern with a fractal dimension of 1.64. The increase in the fractal dimension is related to an increase in the number of branch in the pattern.
\end{abstract}

Keywords: Pattern formation, Ag-In electrodeposit, Fractal dimension, Turing pattern.

\section{INTRODUCTION}

Various kinds of pattern formed in growing fronts in single element electrodeposition such as copper [1], zinc [2], and polypyrrole [3] have been early recognized that they are related to the instability of interface known as the MullinsSekerka instability [4], and that have a physical feature captured well by diffusion-limited aggregates (DLA) [5]. Hence, the surface morphology of the electrodeposit indicated an open and rough appearance, which was far from a dense and smooth surface. The morphological transition between different patterns was also reported to be dependent on the cathode potential [6].

On the other hand, to fabricate thin films of binary alloys, electrodeposition has also been employed in science and technology [7] owing to its simplicity and low cost. For example, electrodeposits of Ag-In binary alloys are known to be a good candidate for a joint alloy because of the proper electrical, mechanical, and anti-diffusive property for microelectronic devices $[8,9]$. In this study, the two kinds of pattern formed in the Ag-In electrodeposits will be reported to have a fractal dimension calculated by a box count method [10]. In contrast to single element electrodeposition, the fractal electrodeposits in the Ag-In thin films have dense and smooth surfaces. In addition, the diagram of morphology for the Ag-In electrodeposits, in which the two kinds of patterns such as the fishbone-like and tree-like pattern exist, shows the dependence of the transition between the patterns on the current density rather than the cathode potential.

As a mechanism of pattern formation in the famous Belousov-Zhabotinsky reaction [11] and chlorite-iodidemalonic acid reaction [12], Turing pattern [11] has been proposed on the basis of the Turing instability referred as to local activation and long-range inhibition. Instead of the Mullins-Sekerka instability and DLA model, from the

*Address correspondence to this author at the Department of Mechanical Systems Engineering, University of the Ryukyus, 1 Senbaru Nishiharacho,Okinawa, 903-0213, Japan; Tel: +81-98-895-8635; Fax: +81-98-8958707; E-mail: saitou@tec.u-ryukyu.ac.jp viewpoint of Turing pattern, we will discuss the formation of the fractal pattern in the Ag-In electrodeposits.

The present paper aims at reporting the fractal pattern having the tree-like and fishbone-like configuration in the Ag-In electrodeposits.

\section{EXPERIMENTAL SETUP}

Experiments for Ag-In electrodeposition were performed using the electrolyte which included the following components (g/l, Kanto Chemical Co. Inc.): $\mathrm{AgNO}_{3}$ 25.5; $\mathrm{K}_{4} \mathrm{Fe}(\mathrm{CN})_{6} \cdot 3 \mathrm{H}_{2} \mathrm{O} \quad 72 ; \quad \mathrm{K}_{2} \mathrm{CO}_{3} \quad 31.3 ; \quad \mathrm{KSCN} \quad 146 ;$ $\mathrm{KNaC}_{4} \mathrm{H}_{4} \mathrm{O}_{6} \cdot 4 \mathrm{H}_{2} \mathrm{O} \quad 59.3 ; \mathrm{KOH} \quad 24.2 ; \operatorname{In}\left(\mathrm{NO}_{3}\right)_{3} \cdot 3 \mathrm{H}_{2} \mathrm{O}$ 5.32. The mixed solution of $\mathrm{AgNO}_{3}, \mathrm{~K}_{4} \mathrm{Fe}(\mathrm{CN})_{6} \cdot 3 \mathrm{H}_{2} \mathrm{O}$, and $\mathrm{K}_{2} \mathrm{CO}_{3}$, was boiled for 30 min and yielded burnt umber precipitates of iron hydroxides. After removal of the iron hydroxide, the remaining components were added into the solution. Thus, the silver cyanide-thiocyanate electrolyte including indium ions was synthesized.

According to the Ag-In phase diagram [13], silver has a solid solubility of 20 atomic wt \% indium at room temperature. Hence, the above-mentioned electrolyte can produce Ag-rich electrodeposits that contain 10 at. wt \% indium.

A poly-crystalline copper (The Nilaco Corp.) and carbon plate (The Nilaco Corp.) were prepared for a cathode and anode electrode, respectively. Their dimensions were $30 \mathrm{~mm}$ long and $10 \mathrm{~mm}$ wide. The copper cathode electrode of 99.9 wt $\%$ purity appeared to be mirror-like. An ultrasonic cleaner with distilled water, acetone, and ethanol in order was used for 10 minutes to clean the electrode. The cathode and anode electrodes were placed parallel in a quiescent electrochemical cell. Potential was applied with an electrical power source through the electrochemical cell connected series to a resistance to change the current density.

The cathode potential was measured with a Luggin capillary with a $\mathrm{Ag} / \mathrm{AgCl}$ electrode in a $\mathrm{KCl}$ solution and was recorded into a digital storage oscilloscope.

The surface morphology of the Ag-In electrodeposit was observed with a laser confocal microscope (Keyence 

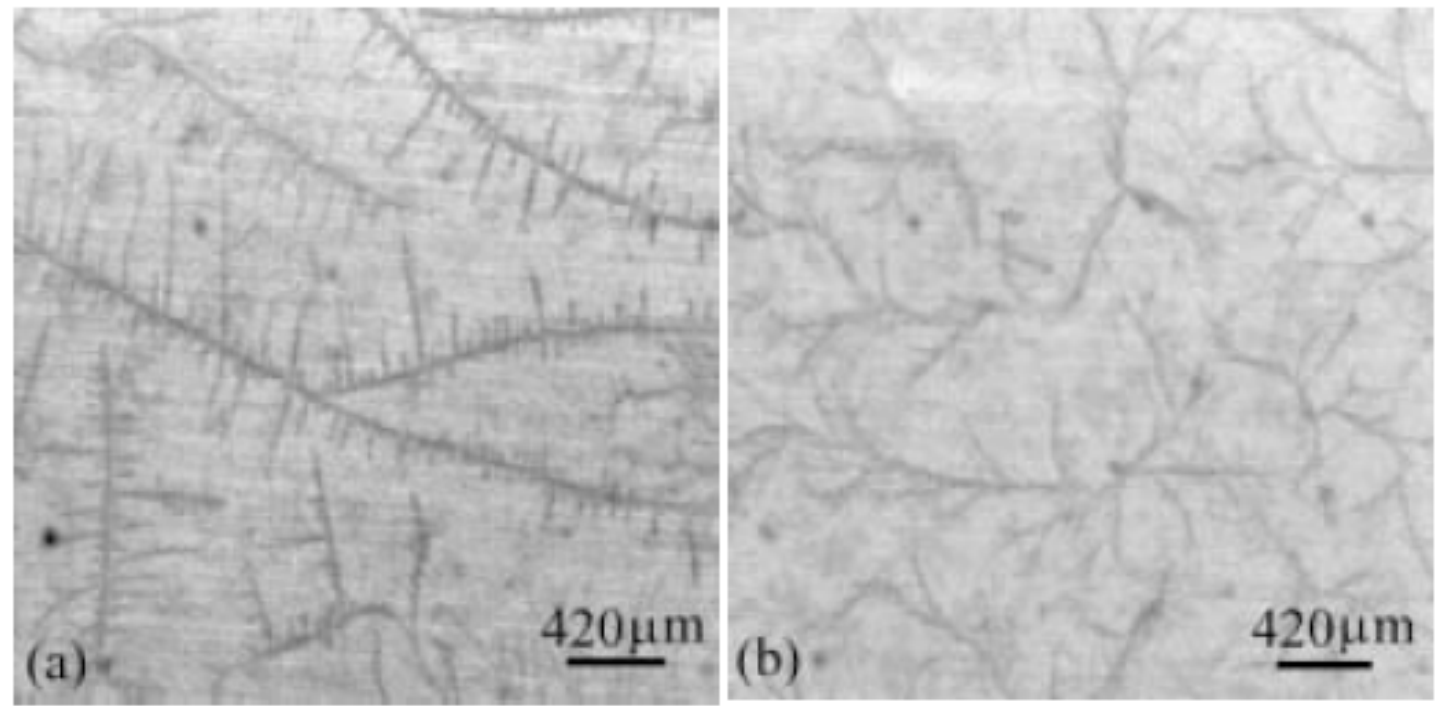

Fig. (1). Typical microscope images of pattern formed in the Ag-In electrodeposits. (a) Fishbone-like patterns formed at a current density of $4.2 \mathrm{~mA} / \mathrm{cm}^{2}$ and a cathode potential of $0.81 \mathrm{~V}$. (b) Tree-like patterns formed at a current density of $5.0 \mathrm{~mA} / \mathrm{cm}^{2}$ and a cathode potential of $0.81 \mathrm{~V}$.

VF7500) having an accuracy of $0.01 \mu \mathrm{m}$ in height and stored in a memory as a digital microscope image with a resolution of $780 \times 564$ pixels.

\section{RESULTS AND DISCUSSION}

In Fig. (1), typical microscope images of pattern formed in the Ag-In electrodeposits at a film thickness of $1.6 \mu \mathrm{m}$ are shown. The brightness difference between the pattern and the other region in the microscope image is due to a difference of indium concentration in the electrodeposits. The patterns in Fig. (1a, b) appear to be fishbone-like and tree-like. In the fishbone-like pattern, a number of small bones diverge from a straight and long backbone. On the other hand, the tree-like pattern is characterized by multiple stems and similar to the pattern generated by the DLA model [5]. However, in contrast to the DLA pattern, the two kinds of pattern are first formed in the electrolyte in the vicinity of the cathode electrode and electrodeposited on the cathode electrode.

The fractal dimension of the pattern, $\mathrm{D}$ is calculated by the box counting method [10] based on the relation, $\mathrm{N} \sim \mathrm{r}^{\mathrm{D}}{ }^{\mathrm{D}}$ where $\mathrm{N}$ is the number of box that includes some part of pattern and $r$ is the box size. The fractal dimensions of the fishbone-like and tree-like pattern are determined from the slopes in Fig. (2) as $1.44 \pm 0.1$ and $1.64 \pm 0.1$, respectively. The larger fractal dimension of the tree-like pattern is related to an increase in the number of branch in the pattern.

Here, we briefly explain the Turing instability. According to the linear theory of Turing pattern in a reaction-diffusion system [11], the Turing instability referred to as local activation and lateral inhibition is given by:

$$
D_{2} / D_{1}>-k_{22} / k_{11}
$$

where $D_{1}$ and $D_{2}$ are the diffusion coefficients of the activator and the inhibitor, and $\mathrm{k}_{11}$ and $\mathrm{k}_{22}$ are the reaction rates for the activator and the inhibitor, respectively. Eq. (1) indicates that the two diffusion coefficients have different values that cause instability in the system. For $\mathrm{k}_{11}>0$, the reactant species act as a catalyst and the other reactant species with $\mathrm{k}_{22}<0$ become an inhibitor. This simply shows that diffusion destabilizes the system. Hence, the tworeactant species are called the activator and the inhibitor in the Turing pattern.

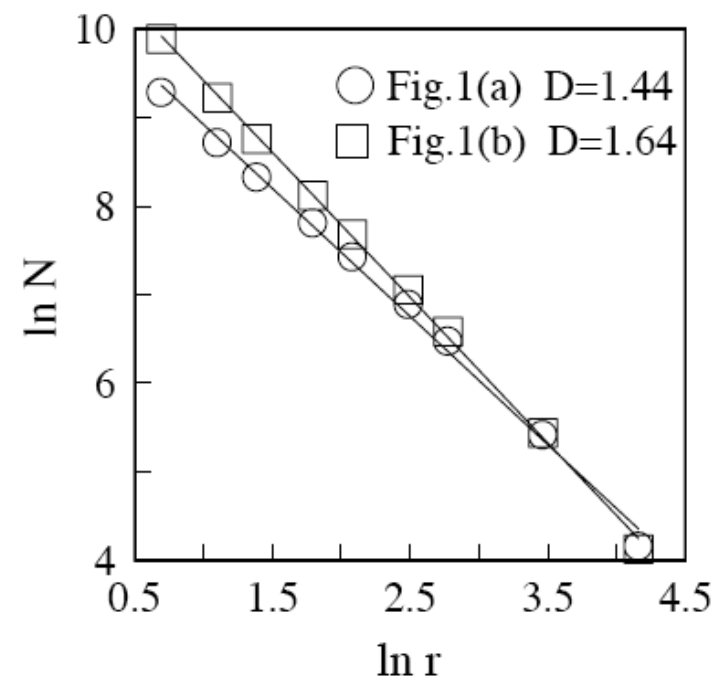

Fig. (2). Fractal dimension determined by the box counting method. The fractal dimensions of the fishbone-like and tree-like pattern become 1.44 and 1.64 .

The Turing instability suggests the presence of the activator and inhibitor for the electrochemical reaction of indium-species, i.e., the formation of the indium rich region adjacent to the indium poor region in the electrolyte. In other binary alloy electrodeposits such as $\mathrm{Ag}-\mathrm{Sb}$, the two regions in which the $\mathrm{Sb}$ concentration is different have been observed [14]. In addition, in order to obtain the fractal pattern in the Ag-In electrodeposits, some sites that behave like a growth origin are needed to occur randomly in the electrolyte. In Fig. (1), the growth origin appears to exist randomly in the Ag-In electrolyte. 


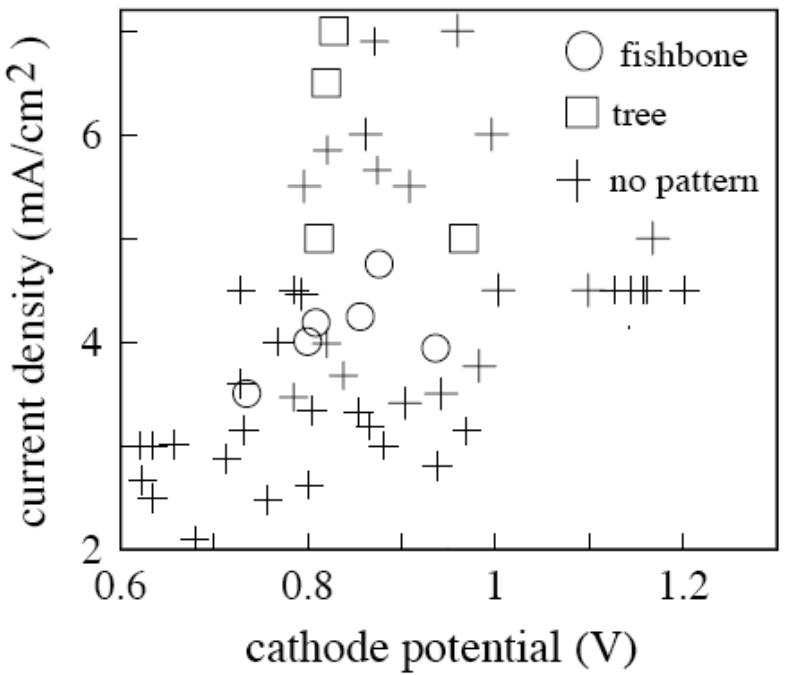

Fig. (3). Diagram of morphology in Ag-In electrodeposition at room temperature. of the electrolyte and affects the transport of ions as shown in Eq. (1).

In order to observe the morphology of pattern, the surface roughness of the Ag-In electrodeposit in Fig. (4), which was measured with the laser confocal microscope, is shown. The standard deviation of the surface roughness becomes $0.18 \mu \mathrm{m}$, which value is approximately equal to that of the copper substrate. There is no difference in height between the pattern and the other region. This indicates that no instability in the interface between the Ag-In electrodeposit and the electrolyte, which is called the Mullins-Sekerka instability, takes place. The pattern and the other region grow together in a direction perpendicular to the electrode. In other words, the electrodeposit has a dense and smooth surface. Hence, the Turing instability is thought to occur in the electrolyte near the cathode electrode.

\section{CONCLUSIONS}

The patterns formed in the Ag-In electrodeposits have the fractal property. The fishbone-like and tree-like pattern have

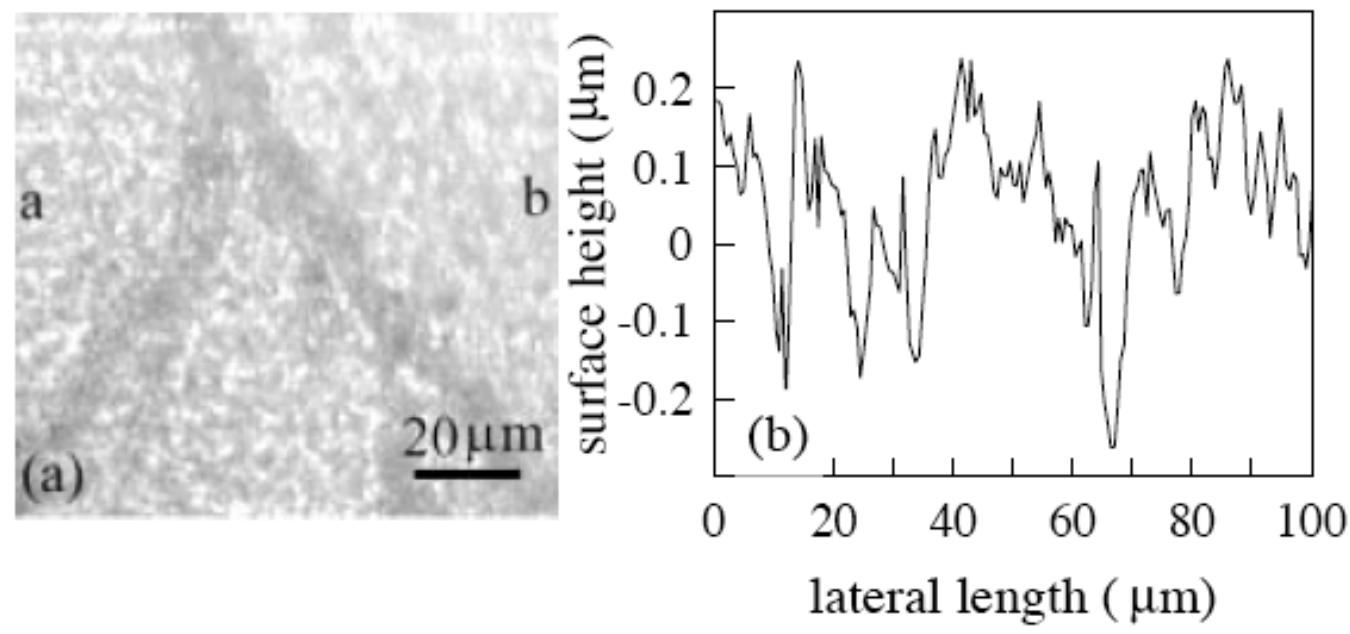

Fig. (4). Surface image and surface height profile measured with the laser confocal microscope. (a) Tree-like pattern formed at a current density of $5.0 \mathrm{~mA} / \mathrm{cm}^{2}$ and a cathode potential of $0.81 \mathrm{~V}$. (b) Surface height profile measured along the scanning line of a-b in (a).

The electrochemical reactions for the formation of pattern in Ag-In electrodeposition are at present unclear. However, the charge transfer reaction of Ag complex agents in the electrolyte becomes:

$$
A g(C N)_{2} \mathrm{CNS}_{\varphi}^{-(\varphi+1)}+e^{-} \rightarrow A g+2 C N^{-}+\varphi C N S^{-} .
$$

where $\phi$ has a value between 1 and 2 . We therefore construct a diagram of morphology in Ag-In electrodeposition to investigate the presence of pattern at a current density and cathode potential. The electrochemical species that behave as the activator and inhibitor will be generated in the electrolyte and the pattern is electrodeposited on the cathode electrode. In Fig. (3), the patterns are seen to form within a narrow region in the diagram. The transition from the fishbone-like pattern to the tree-like pattern is shown to be dependent on the current density rather than the cathode potential. The cathode potential is thought to influence the instability of surface within a framework of the Mullins-Sekerka instability [3]. On the other hand, from the viewpoint of the Turing pattern, the current density changes the components the fractal dimensions of 1.44 and 1.64. In addition, the patterns are found to be formed within a narrow region in the morphology diagram.

\section{REFERENCES}

[1] Brady, R.M.; Ball, R.C. Fractal growth of copper electrodeposits, Nature, 1984, 309, 225-9.

[2] Matsushita, M.; Sano, M.; Hayakawa, Y.; Honjo, H. Sawada, Fractal structure of zinc metal leaves grown by electrodeposition, Y. Phys. Rev. Lett., 1984, 53, 286-9.

[3] Kaufman, J.H.; Nazzal, A.I.; Melroy, O.R.; Kapitulnik, A. Onset of fractal growth: Statistics and dynamics of diffusion-controlled polymerization, Phys. Rev. B, 1987, 35, 1881-90.

[4] Mullins, W. W. ; Sekerka, R. F., The stability of a planar interface during solidification of adilute binary alloy. J. Appl. Phys., 1964, $35,444-51$.

[5] Witten, T.A.; Sander, L.M. Diffusion-limited aggregation, a kinetic critical phenomenon. Phys. Rev. Lett., 1981, 47, 1400-3.

[6] Sagués, F. ; L-Salvans, M. Q. ; Claret, J., Growth and forms in quasi-two-dimensional electrocrystallization. Phys. Reports, 2000, 337, 97-115.

[7] Pandey, R.K.; Sahu, S.N.; Chandra, S. Handbook of Semiconductor Electrodeposition, Marcel Dekker, New York, 1996. 
[8] Lee, C.C.; So, W.W. High temperature silver-indium joints manufactured at low temperature. Thin Solid Films, 2000, 366, 196-201.

[9] Bukaluk, A. ; Trzciński, M. ; Okulewicz, K., Electron spectroscopy studies of surface In-Ag alloy formation on the tungsten surface. Appl. Surf. Sci., 2008, 254, 4263-7.

[10] Vicsek, T. Fractal Growth Phenomena. World Scientific, London, 1989.
[11] Kapral, K.; Showalter, K. Chemical Waves and Patterns. Kluwer Academic Press, Dordrecht, 1995, pp. 367-9.

[12] DeKepper, P.; Epstein, I.R.; Kustin, K.; Orbán, M. Batch oscillations and spatial wave patterns in chlorite oscillating systems. J. Phys. Chem., 1982, 86, 170-1.

[13] Massalski, T.B. Binary Alloy Phase Diagram, ASM Intl., Ohio, 1996, pp. 47-8

[14] Saitou, M. ; Y. Fuoka, Y., Stripe pattern formation in Ag-Sb coelectrodeposition. Electrochim. Acta, 2005, 50, 5044-9.

Received: April 10, 2009

Revised: May 29, 2009

Accepted: June 3, 2009

(C) Saitou et al.; Licensee Bentham Open.

This is an open access article licensed under the terms of the Creative Commons Attribution Non-Commercial License (http: //creativecommons.org/licenses/by$\mathrm{nc} / 3.0 /$ ) which permits unrestricted, non-commercial use, distribution and reproduction in any medium, provided the work is properly cited. 